\title{
Effects of Ethanol, Diphenhydramine, and Triazolam after a Nap
}

Timothy Roehrs, Ph.D., Deirdre Claiborue, B.S., Michele Knox, B.S., and Thomas Roth, Ph.D.

This study assessed the capacity of a 60-minute nap to reverse the sedating and performance-disruptive effects of ethanol, triazolam, and Diphenhydramine. Twelve healthy, young men received (at 0800 to 0830 ) $.6 \mathrm{~g} / \mathrm{kg}$ ethanol and a placebo pill, .25 $\mathrm{mg}$ triazolam and ethanol placebo, $50 \mathrm{mg}$ Diphenhydramine and ethanol placebo, and a placebo pill and ethanol placebo on each of 2 days in a Latin Square design. On one treatment day (at 0900 hour) subjects were allowed a 60-minute nap and on the other a sleep latency test (no nap). Then, sleep latency was tested (by Multiple Sleep Latency Test [MSLT] at
$1100,1300,1500$, and 1700) and divided attention performance assessed (at 1130). The nap reversed the sedative effects of ethanol and Diphenhydramine and reduced those of triazolam (on MSLT). The nap reduced the performance-disruptive effects of ethanol and Diphenhydramine but not those of triazolam. Given the differing neurobiological mechanisms of these drugs, the data suggest that some of their effects are nonspecific and result from activation of sleep mechanisms that a nap can reverse. [Neuropsychopharmacology 9:239-245, 1993]
KEY WORDS: Ethanol; Diphenhydramine; Triazolam; Multiple Sleep Latency Test

Various drugs of differing classes have sedative and performance-disruptive effects. Daytime studies using standard polysomnographic methods to objectively document sedative effects (the Multiple Sleep Latency Test [MSLT]), have shown that $\mathrm{H}_{1}$ antihistamines shorten sleep latency in a systematic dose-related fashion (Nicholson and Stone 1986; Roehrs et al. 1984). Benzodiazepines also hasten sleep onset after daytime administration, again in a dose-related manner (Roehrs et al. 1988). Finally, ethanol also has a dose-related effect on sleep latency as measured by the MSLT (Zwyghuizen-Doorenbos et al. 1988). All these drugs also disrupt

From the Henry Ford Hospital, Sleep Disorders and Research Center, Detroit, Michigan.

Address reprint requests to: Timothy Roehrs, Ph.D., Henry Ford Hospital, 2921 West Grand Boulevard, Detroit, Michigan 48202.

Received February 9, 1993; revised July 7, 1993; accepted July 7, 1993. performance on various psychomotor, cognitive, and memory tasks, and the disruptive effects associated with some have been found to relate to the hypnotic potency of the specific drug and dose (Roehrs et al. 1993; Merlotti et al. 1992).

Although they share sedative and performancedisruptive effects, these drugs are thought to produce their effects through quite different neurobiologic mechanisms. The benzodiazepines are known to facilitate chloride conductance at the GABA receptor complex (Mendelson et al. 1984). Ethanol also alters chloride conductance, but its sedating effects appear to be due to enhancement of calcium uptake (Mendelson 1989). Finally, the antihistamines act at central histamine receptors, blocking the action of histamine, now considered a waking amine (Schwartz et al. 1982).

The common sedative and performance-disruptive effects of these drugs, given their differing neurobiologic mechanisms, suggest that these effects are nonspecific and may result from activation of sleep mechanisms. If the sedation is due to activation of a sleep drive, then sleep per se should reverse these effects. One previous study of the effects of a 60 -minute nap following drug administration showed that the nap re- 
duced the sedative and performance-disruptive effects of a moderate ethanol dose (Roehrs et al. 1990). The question arises as to whether a nap will similarly reverse or reduce the sedative effects associated with a benzodiazepine and a $\mathrm{H}_{1}$ antihistamine.

\section{METHODS}

\section{Subjects}

The subjects were 12 healthy young men, aged 21 to 35 years (mean $24.3 \pm 2.4$ years). The subjects did not smoke, currently use licit or illicit drugs (confirmed by a urine drug screen), and reported drinking an average of 1 to 14 alcoholic drinks per week. They were in good physical and mental health based on their medical and drug-use histories, a physical examination, and laboratory analysis of blood and urine samples. They reported normal sleep habits with nocturnal sleep latencies of $\mathbf{3 0}$ minutes or less, no sleep-maintenance problems, no habitual daytime napping, a regular sleep schedule with bedtime and time of arising not varying by more than 2 hours, and no clinical evidence of sleep disorders. Each signed an informed consent (approved by the Institutional Review Board) and was paid for participation.

\section{Procedure}

The subjects qualifying on the initial screening were asked to come to the sleep laboratory for a one-night screening and laboratory adaptation. Each reported 1 hour prior to their usual bedtime, and electrodes were attached at standard Rechtschaffen and Kales (1968) placements to record electroencephalograms (EEGs, central and occipital), electrooculograms (EOGs, left and right outer canthi), and an electromyogram (EMG, submental) with the addition of a tibialis EMG to detect leg movements and a nasal/oral thermistor to detect respiratory disturbance. They went to bed for 8 hours while their sleep was monitored. Those with a sleep efficiency (sleep time per time in bed) of $85 \%$ or less and any evidence of leg movements or respiratory disturbance were excluded.

The following day the MSLT was administered at $1000,1200,1400$, and 1600 according to standard procedures (Carskadon et al. 1986). For each latency test subjects went to bed in a dark, quiet room and were instructed to try to fall asleep while EEGs (always including the occipital), EOGs, and the EMG (submental) were monitored. Subjects were awakened after 1 minute of unambiguous stage 1 sleep, the first signs of stage 2 or rapid-eye movement (REM) sleep, or 20 minutes of continuous wake according to the standards of Rechtschaffen and Kales (1968). Subjects with a mean daily sleep latency (minutes to first epoch of sleep) of 10 minutes or more were included.

Qualified subjects underwent each of four drugethanol treatment conditions lasting 2 days (a total of 8 days), with conditions presented in a repeated measures Latin Square design. The treatment conditions were $.6 \mathrm{~g} / \mathrm{kg}$ ethanol and a placebo pill, $50 \mathrm{mg}$ Diphenhydramine and ethanol placebo, .25 $\mathrm{mg}$ triazolam and ethanol placebo, and a placebo pill and ethanol placebo. The ethanol was prepared in a 1:4 ratio with 80 proof vodka added to tonic water, and the ethanol placebo consisted of the tonic water with three drops of vodka floated on the surface for gustatory and olfactory cues. The drugs and placebos were presented in similar looking opaque capsules. Each condition included a napand a no-nap day (the 2 days of each condition) that were counterbalanced within each condition. The conditions were presented once a week on the same 2 weekdays.

Prior to each experimental day subjects spent 8 hours in bed (2300-0700), while sleep was monitored with an actigraph (Levine et al. 1986). Subjects arose at 0700 , used the toilet, and ate a small breakfast consisting of a noncaffeinated beverage and a roll. They then received the pill at 0800 and consumed the ethanol or placebo drink between 0800 and 0830 , pacing their drinking over the 30 minutes. At 0900 subjects went to bed for a 60-minute nap (nap condition) or had a sleep latency test (no-nap condition). In all conditions latency tests were then conducted at $1100,1300,1500$, and 1700 as described in the screening. Subjects were allowed to leave the laboratory after the last latency test and were required to return to the laboratory 1 hour before bedtime on the next study day.

The pharmacokinetics of the drugs used in this study are known to differ. Blood samples were not drawn in this study to characterize plasma concentration curves. Plasma concentration curves following a drug administration at 0900 (at 0800 drug administration was used in this experiment to provide for the 60minute nap) for the doses of ethanol, triazolam, and Diphenhydramine used in this experiment can be found in a previous paper (Roehrs et al. 1993). Because some studies have found differences in breath-ethanol concentration (BEC) as a function of sleep versus wake, BEC was measured at approximately 0900, 1100, 1300, 1500, and 1700 just prior to each of the latency tests (or the 60-minute nap). The BEC measurement was conducted every day, regardless of whether ethanol or ethanol placebo had been administered. It was measured using an Alcotest 7010 National Draeger Breathalyzer.

At 1130 a 15-minute divided attention performance test was administered. Each subject was trained on the divided attention test during the screening day. The test was presented on a video screen controlled by an 
Apple IIc computer. During the test, subjects were seated in front of the video screen with the task of tracking a moving target using a joystick that was maneuvered with the preferred hand. At the same time, subjects responded to the appearance of a target stimulus (a white circle) in the center or in the periphery of the screen by pressing a button located adjacent to the joystick. Reaction times (RT) to the central stimulus, the peripheral stimulus, and the tracking deviations were recorded.

During each day, subjects were instructed to refrain from any napping and caffeine use and were continuously monitored throughout the day to ensure compliance. Alcohol and drug use other than that required in the study was also prohibited. Subjects were told that urine samples would be collected during the study and tested for the presence of unauthorized use of drugs.

The MSLT was scored for sleep latency according to the standards of Rechtschaffen and Kales (1968) by scorers unaware of the treatment condition. Sleep latency was defined as time from lights out to the first epoch of sleep. Sleep latency (mean of the 1100, 1300, 1500 , and 1700 tests) and the three performance measures described were analyzed for drug and nap effects, using the general linear models multivariate analysis of variance (SAS Institute). Conservative $p$-levels of $p$ $<.05$ or higher, corrected by the Huynh-Feldt procedures, were used to determine significance. Each drug condition was compared to the placebo condition in a separate two-factor (drug and nap) repeated measures analysis, as differences among the three drugs were not of direct interest. Analyses of time effects for each drug versus placebo comparison were also conducted by adding time of test $(1100,1300,1500$, and 1700) as a third repeated measures variable.

In the post hoc comparisons of the four drug and nap treatment combinations, a result showing that the drug-nap condition did not differ from the placebo-nonap condition, whereas the drug-no-nap condition did differ from the placebo-no-nap condition was interpreted as a nap-related reversal of sedative or performance-disruptive effects of the drug. An attenuation of drug effects by the nap was interpreted when the pattern of results showed that the drug-nap condition was intermediate to the placebo-no-nap and the drug-no-nap conditions.

\section{RESULTS}

\section{Prior Nocturnal Sleep}

The sleep efficiency, defined as percentage of inactive time per time in bed (determined by actigraph) prior to each of the eight treatment days, is presented in Table 1 . Sleep efficiencies in these healthy normals ranged from $88.0 \%$ to $92.7 \%$. There were no significant differences in efficiencies among the four treatment conditions or between the nap and no-nap days of each treatment.

\section{Breath-Ethanol Concentration}

The BEC did not differ significantly on the nap and nonap days, and the data are combined. Average BEC over both days at 30 minutes after consumption (0900) was $.044 \% \pm .014 \%$. At 1100 the BEC was $.037 \% \pm .013 \%$, it declined to $.014 \% \pm .013 \%$ at 1300 , and reached zero at 1500 . There was a significant time of test effect $(F=$ $67.76, p<.001$ ), with the BEC at 0900 differing from that at 1300,1500 , and 1700 and the BEC at 1100 similarly differing from that of the last three tests. Finally, the BEC at 1300 differed from those at 1500 and 1700.

\section{Nap Effects on Mean Sleep Latency (MSLT)}

The mean daily sleep latency with and without a nap after ethanol consumption is compared to the placebo conditions in Figure 1. In the analysis comparing the four drug and nap combinations there was an overall condition effect $(F=11.21, p<.001)$. Latency in the ethanol-no-nap condition was shorter than that of the placebo-no-nap condition $(p<.001)$, reflecting the sedating effect of ethanol. The latency of the ethanolnap condition did not differ from that of the placebo-nonap condition, but it did differ from that of the ethanol-no-nap condition $(p<.001)$, being longer and reflecting a reversal of the sedating effect of ethanol.

Diphenhydramine mean daily sleep latency with and without a nap is compared to that of the placebo conditions in Figure 2. In the comparison of four treatment-nap combinations a main effect of conditions was found $(F=10.79, p<.001)$. As for ethanol, a sedating effect of Diphenhydramine was seen as shown in the significant Diphenhydramine-no-nap versus pla-

Table 1. Nocturnal Sleep Efficiency

\begin{tabular}{lcccc}
\hline & Placebo & Ethanol & Diphenhydramine & Triazolam \\
\hline Nap day & $92.7 \pm 2.7$ & $90.3 \pm 6.9$ & $88.3 \pm 8.0$ & $90.5 \pm 5.4$ \\
No-nap day & $88.0 \pm 12.4$ & $90.3 \pm 3.9$ & $91.3 \pm 4.1$ & $89.1 \pm 8.0$ \\
\hline
\end{tabular}

Data are means \pm SD. 


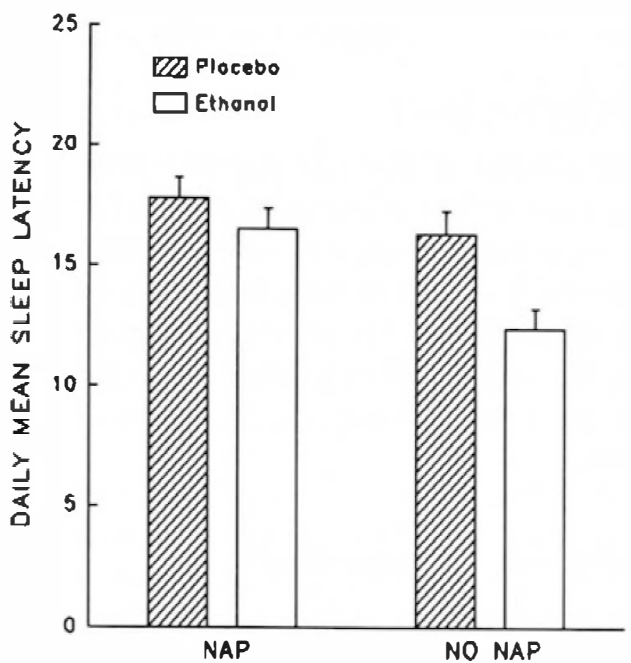

Figure 1. Mean daily sleep latency (minutes) after placebo or $.6 \mathrm{~g} / \mathrm{kg}$ ethanol and a 60 -minute nap or no nap.

cebo-no-nap comparison $(p<.003)$. The nap reversed this sedating effect, as the Diphenhydramine-nap condition did not differ from the placebo-no-nap condition and did differ from the Diphenhydramine-no-nap condition $(p<.01)$.

In Figure 3 the mean daily sleep latency following triazolam with and without a nap is compared to that of the placebo conditions. The main effect of conditions was significant $(F=24.65, p<.001)$. The sedating effect of triazolam was seen in the significant triazolam-nonap versus placebo-no-nap comparison $(p<.001)$. The nap reduced these sedating effects, as the triazolamnap condition differed from both the placebo-no-nap condition and the triazolam-no-nap condition $(p<$ $.001)$, being intermediate to the two.

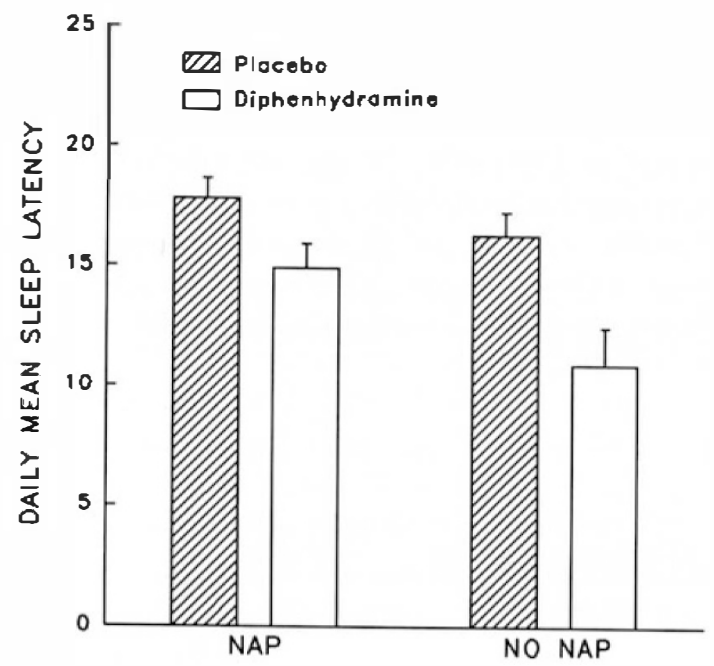

Figure 2. Mean daily sleep latency (minutes) after placebo or $50 \mathrm{mg}$ Diphenhydramine and a 60 -minute nap or no nap.

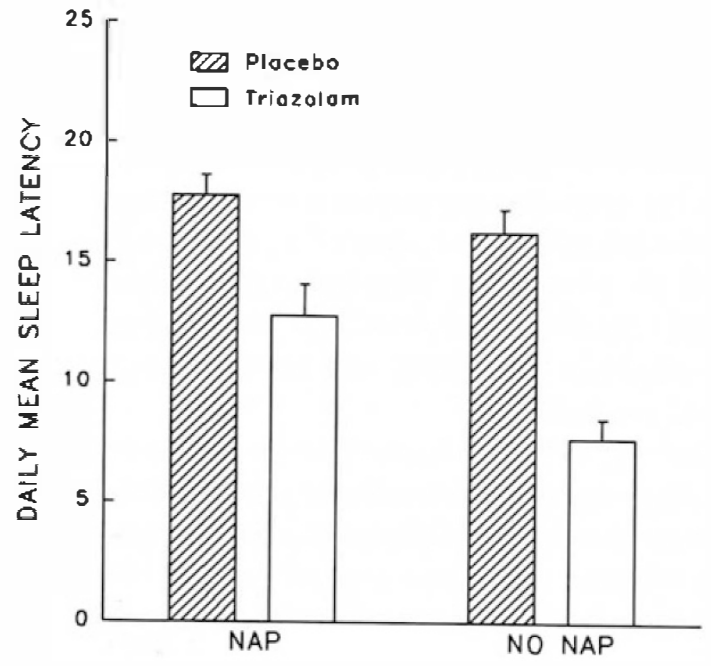

Figure 3. Mean daily sleep latency (minutes) after $.25 \mathrm{mg}$ triazolam and a 60 -minute nap or no nap.

\section{Time Effects on Sleep Latency (MSLT)}

Sleep latency on each test $(0900,1100,1300,1500$, and 1700 ) is presented in Table 2 . On the table the nap versus no-nap conditions are combined, as there were no significant triple interactions (i.e., nap by time by drug). In the analysis of ethanol effects a significant time $(F=5.00, p<.006)$ and time by ethanol interaction $(F=$ $3.27, p<.03)$ were found. Ethanol differed from placebo on the first two tests only $(p<.05)$. In the analysis of Diphenhydramine effects a significant time $(F=8.94$, $p<.001)$ and time by Diphenhydramine interaction $(F=$ $4.74, p<.01)$ were found. Drug effects were found on the first three tests $(p<.05)$. Finally, for triazolam a significant time $(F=16.22, p<.0001)$ and time by triazolam interaction $(F=13.91, p<.0001)$ were found. Drug effects after triazolam appeared on the first three tests $(p<.05)$.

\section{Nap Effects on Psychomotor Performance (Divided Attention)}

The divided attention measures for each drug condition with and without a nap are presented in Table 3. In the ethanol condition the comparison of the effects of the nap on tracking showed a main effect of condition $(F=2.95, p<.04)$. The post hoc comparisons showed that the ethanol-no-nap condition differed from the placebo-no-nap condition $(p<.01)$, reflecting the disruptive effects of ethanol on this performance measure. The ethanol-nap condition did not differ from the placebo-no-nap condition or from the ethanol-nonap condition (i.e., was intermediate), suggesting that the nap reduced the ethanol effects.

As with tracking, main effects of condition in the 
Table 2. Sleep Latency on Each Test in Each Condition

\begin{tabular}{lrrrrr}
\hline & \multicolumn{5}{c}{ Time (hours) } \\
\cline { 2 - 6 } & \multicolumn{1}{c}{$\mathbf{0 9 0 0}$} & \multicolumn{1}{c}{$\mathbf{1 1 0 0}$} & \multicolumn{1}{c}{$\mathbf{1 3 0 0}$} & $\mathbf{1 5 0 0}$ & \multicolumn{1}{c}{$\mathbf{1 7 0 0}$} \\
\hline Placebo & $16.5 \pm 5.29$ & $16.7 \pm 4.62$ & $16.9 \pm 2.88$ & $16.0 \pm 3.95$ & $18.6 \pm 2.42$ \\
Ethanol & $14.5 \pm 4.73$ & $11.2 \pm 3.12$ & $15.7 \pm 4.43$ & $15.7 \pm 4.75$ & $17.4 \pm 3.39$ \\
Diphenhydramine & $12.8 \pm 6.69$ & $9.9 \pm 5.47$ & $11.5 \pm 5.48$ & $15.1 \pm 3.72$ & $17.4 \pm 3.18$ \\
Triazolam & $5.8 \pm 6.34$ & $4.0 \pm 1.82$ & $9.4 \pm 5.05$ & $14.0 \pm 4.44$ & $16.1 \pm 4.59$ \\
\hline
\end{tabular}

Data are means \pm SD.

ethanol analysis were found on peripheral reaction time $(F=4.09, p<.03)$ and central reaction time $(F=3.19$, $p<.04)$. Disruptive effects were seen in that the ethanol-no-nap condition differed from the placebo-no-nap condition for each measure $(p<.005$ and $p<.01$, respectively). The nap reduced this effect on central reaction time (ethanol-nap condition was intermediate between the placebo-no-nap and ethanol-no-nap conditions) but had no effect on the peripheral reaction time (ethanol-nap condition differed from placebo-no-nap condition, $p<.03$, did not differ from the ethanol-nonap condition).

On the Diphenhydramine analysis of nap effects, main effects of condition were found for tracking ( $F=$ 5.04, $p<.005)$, peripheral reaction time $(F=4.35, p<$ $.03)$, and central reaction time $(F=3.23, p<.04)$. Diphenhydramine was associated with a disruption of each parameter (Diphenhydramine-no-nap condition differed from placebo-no-nap condition, $p<.008, p<$ $.01, p<.04$, respectively). The nap reduced this effect on central reaction time (Diphenhydramine-nap condition did not differ from placebo-no-nap condition) but had no effect on tracking or peripheral reaction time.

Finally, in the triazolam analysis of nap effects condition main effects were found for tracking $(F=5.82$, $p<.003)$, peripheral reaction time $(F=7.91, p<.001)$, and central reaction time $(F=7.90, p<.001)$. Triazolam was associated with a disruption of each parameter (triazolam-no-nap condition differed from placebo-no-nap condition, $p<.01, p<.009, p<.007$, respectively). The nap failed to reverse or reduce the disruptive effects of triazolam (for each measure triazolam-nap condition differed from placebo-no-nap condition, $p<.006, p<.001, p<.001$, respectively, and did not differ from the triazolam-no-nap condition).

\section{Drug Effects on Nap Sleep}

Table 4 presents the sleep stage parameters on the 60 minute nap for each of the four drug conditions. Comparing the drug conditions, a main effect of condition was found for total sleep time $(F=8.38, p<.02)$ with the triazolam condition differing from placebo $(p<.05)$. The combination of percent wake and stage 1 sleepalso showed condition effects $(F=3.39, p<.04)$, again with triazolam differing from placebo $(p<.05)$. Finally, for percentage stage $3 / 4$ sleep there was a condition effect $(F=3.08, p<.05)$, and on the post hoc testing Diphenhydramine differed from placebo.

\section{DISCUSSION}

The 60-minute nap reversed the sedative effects (as measured by MSLT) of ethanol $(.6 \mathrm{~g} / \mathrm{kg})$ and Diphenhydramine $(50 \mathrm{mg})$ and reduced the effects of triazolam $(.25$ $\mathrm{mg}$ ). The 60-minute nap reduced the performancedisruptive effects of ethanol and Diphenhydramine but not those of triazolam. In part, the differential hypnotic effects of these drugs at the doses studied can explain

Table 3. Divided Attention Performance

\begin{tabular}{lcccc}
\hline & Placebo & Ethanol & Diphenhydramine & Triazolam \\
\hline Tracking & & & & \\
$\quad$ Nap day & $23.3 \pm 6.4$ & $21.0 \pm 5.0$ & $25.4 \pm 6.4$ & $30.0 \pm 10.0$ \\
$\quad$ No-nap day & $19.5 \pm 3.6$ & $23.0 \pm 5.0$ & $25.7 \pm 7.5$ & $35.7 \pm 20.4$ \\
Central RT & & & \\
$\quad$ Nap day & $0.53 \pm .10$ & $0.52 \pm .10$ & $0.50 \pm .10$ & $0.71 \pm .20$ \\
$\quad$ No-nap day & $0.46 \pm .10$ & $0.56 \pm .10$ & $0.58 \pm .20$ & $0.80 \pm .40$ \\
Peripheral RT & & & $0.55 \pm .10$ & $0.75 \pm .30$ \\
$\quad$ Nap day & $0.56 \pm .10$ & $0.60 \pm .20$ & 0.50 \\
$\quad$ No-nap day & $0.47 \pm .10$ & $0.61 \pm .20$ & $0.62 \pm .20$ & $0.84 \pm .50$ \\
\hline
\end{tabular}

Data are means $\pm \mathrm{SD}$. 
Table 4. Nap-Sleep Parameters

\begin{tabular}{lcccc}
\hline & Placebo & Ethanol & Diphenhydramine & Triazolam \\
\hline Percent sleep & $67.7 \pm 31.9$ & $57.7 \pm 27.5$ & $69.8 \pm 21.4$ & $89.5 \pm 12.4$ \\
Percent stage 1 & $49.1 \pm 41.1$ & $42.6 \pm 29.6$ & $38.9 \pm 35.3$ & $30.3 \pm 22.8$ \\
Percent stage 2 & $48.6 \pm 39.6$ & $54.6 \pm 29.3$ & $54.2 \pm 32.8$ & $59.6 \pm 26.9$ \\
Percent stage 3/4 & $0.0 \pm 0.0$ & $0.0 \pm 0.0$ & $6.9 \pm 13.1$ & $3.7 \pm 11.2$ \\
Percent stage REM & $2.3 \pm 6.0$ & $2.8 \pm 5.1$ & $0.0 \pm 0.0$ & $6.4 \pm 14.2$ \\
Percent wake + 1 & $81.4 \pm 64.7$ & $84.9 \pm 52.0$ & $69.0 \pm 34.7$ & $40.8 \pm 31.1$ \\
Entries to wake +1 & $96.3 \pm 33.4$ & $6.0 \pm 1.9$ & $0.7 \pm 0.8$ & $1.6 \pm 1.6$ \\
\hline
\end{tabular}

Data are means \pm SD.

the differences in the effectiveness of the nap in reversing or reducing the drug effect. In Figure 4 the differing hypnotic effects of the drugs are compared to illustrate this point. Triazolam produced the greatest reduction in sleep latency, and the nap was least effective in reversing the effects of triazolam. On the other hand, ethanol was associated with the smallest reduction in sleep latency, and the nap was most effective in reversing the effects of ethanol.

The relative potency of triazolam to the other drugs also is illustrated by the drug effects on nap sleep. Triazolam among the active drugs increased sleep on the 60-minute nap and also reduced the percentage of light sleep (stage 1). Numerically, the Diphenhydramine values fell between those of triazolam and placebo. Diphenhydramine also increased percentage stage $3 / 4$ sleep, which was not seen with triazolam. But in allnight studies the benzodiazepines have been consistently shown to reduce stage $3 / 4$ sleep. Consequently, with triazolam one would expect to find no effect or even a reduction in stage $3 / 4$ on the nap.

An alternative viewpoint regarding the hypothesis that triazolam effects were not reversed because of the relatively greater hypnotic effects of triazolam can be mentioned. It could be argued that the greater

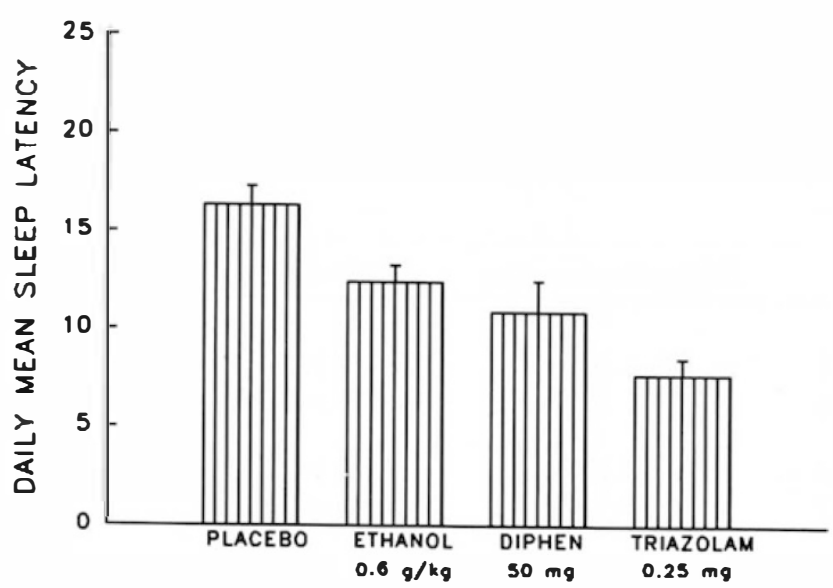

Figure 4. Mean daily sleep latency (minutes) after placebo, ethanol $.6 \mathrm{~g} / \mathrm{kg}$, Diphenhydramine $50 \mathrm{mg}$, and triazolam $.25 \mathrm{mg}$. amount of sleep on the 60-minute nap with triazolam should produce improved performance and alertness. But, the difference in sleep time between placebo and triazolam was 12 minutes (41 vs. 53 minutes). In 24 hour-sleep-deprived normals a 15-minute nap failed to improve sleepiness or alertness, but 30-to 120-minute naps did (Lumley et al. 1986). The subjects in this study were fully rested, having had 7 to 8 hours of sleep the night before each daytime assessment. It is unlikely that the increased nap sleep in the triazolam condition would result in detectable performance effects.

The point must be made that conclusions regarding the effects of the drugs and the capacity of the 60minute nap to reverse those effects in this study are limited by the fact that concurrent plasma concentrations of triazolam and diphenhydramine were not obtained. In a previous study plasma concentration was measured, and peak concentrations of triazolam and Diphenhydramine at the doses used in this study were seen 3.5 hours after administration at 0900 (Roehrs et al. 1993). The performance testing in this study began 3.5 hours post drug administration, which was probably close to the peaks of triazolam and Diphenhydramine. The peak ethanol concentration as measured by the BEC occurred much earlier, sometime between 0900 and 1100 .

The effectiveness of the nap in reversing the effects of ethanol replicates the findings of a previous study. In that study $.6 \mathrm{~g} / \mathrm{kg}$ ethanol was administered, and a peak BEC of .04\% was achieved. A 60-minute nap completely reversed the sedating effects of ethanol as measured by the MSLT (Roehrs et al. 1990). That study did not include a performance assessment, as did the present study. In this study, with similar peak BECs (.044\%), performance effects were reduced but not reversed. The reduction in performance effects, as opposed to a complete reversal of MSLT effects as found in the previous and present study, may reflect two factors. First, the MSLT may be a more sensitive measure with less variability, which is thus capable of more readily detecting the nap effects. Second, there may be components necessary to performing a divided attention task that are affected by ethanol but are not associated with sleepiness and therefore are not improved by the nap. 
The modest effect of the nap in reducing the effects of $.25 \mathrm{mg}$ triazolam can be compared to the findings of another study, in which caffeine was used to counteract the disruptive effects of triazolam $.50 \mathrm{mg}$ on performance measures (Roehrs et al. 1988). Unfortunately, the triazolam doses differed between these two studies. But, in that study both $4 \mathrm{mg} / \mathrm{kg}$ and $8 \mathrm{mg} / \mathrm{kg}$ caffeine did not reduce the sedative and amnestic effects of triazolam 2 hours after administration. Even 4.5 hours postadministration $4 \mathrm{mg} / \mathrm{kg}$ caffeine was unable to reduce the effects of triazolam, although the $8-\mathrm{mg} / \mathrm{kg}$ dose completely reversed the effects. It would be interesting to compare the potency of caffeine to that of a nap in reversing sedative drug effects.

It is interesting to consider the sedative and performance-disruptive effects of Diphenhydramine $50 \mathrm{mg}$ relative to those of ethanol and triazolam. Diphenhydramine in the $50 \mathrm{mg}$ dose appeared to be intermediate to ethanol and triazolam in this study. These results can be discussed in the context of the only previous study to have directly compared the sedative effects of an antihistamine to that of a benzodiazepine (Nicholson and Stone 1986). That study found that triprolidine $2.5 \mathrm{mg}$ produced similar sedative effects to those of $\mathrm{Di}$ azepam $5 \mathrm{mg}$, although there were some differences in the time course of the sedative effects. Triazolam .25 mg would generally be considered more equivalent to $10 \mathrm{mg}$ Diazepam in terms of sedative effects. How the sedative effects of triprolidine might compare to Diphenhydramine is not known.

The mechanism by which the sedative effects of these drugs is reversed is not certain. We have hypothesized that all these drugs are activating homeostatic sleep mechanisms, that is, a sleep drive. The neurobiology of the sleep drive is not known. Whatever its neurobiology, it seems clear that the central nervous system (CNS) has the capacity to monitor the state of sleepiness or alertness. The concurrent status of sleepiness or alertness alters the sensitivity of the CNS to sedating drugs. In addition, whether the sleep drive is activated by sleep deprivation or a sedative drug, sleep per se can reduce or reverse the sleepiness. On the other side, the accumulation of sleep (i.e., increased alertness) prior to administration of a sedative drug can blunt the sedative effects of that drug. This has been demonstrated in humans with ethanol (Roehrs et al. 1989) and recently in animals with the benzodiazepine triazolam (Edgar et al. 1991). In each study, sleep time was increased prior to the administration of the drug, and the sedative effects of the drug seen after normal amounts of sleep were not observed.

\section{ACKNOWLEDGMENT}

This work was supported by National Institutes of Health (NIAAA) Grant No R01 AA07147 awarded to Dr. T. Roehrs.

\section{REFERENCES}

Carskadon MA, Dement WC, Mitler M, Roth T, Westbrook P, Keenan S (1986): Guidelines for the Multiple Sleep Latency Test (MSLT): A standard measure of sleepiness. Sleep 9:519-524

Edgar DM, Seidel WF, Martin CE, Sayeski PP, Dement WC (1991): Triazolam fails to induce sleep in suprachiasmatic nucleus-lesioned rats. Neurosci Lett 125:125-128

Levine B, Roehrs T, Zorick F, Roth T (1986): Actigraphic monitoring and polygraphic recording in determination of sleep and wake. Sleep Res 15:247-xxx

Lumley M, Roehrs T, Zorick F, Lamphere J, Roth T (1986): The alerting effects of naps in sleep-deprived subjects. Psychophysiology 23:403-408

Mendelson W (1989): Is sleep induction by ethanol mediated by alteration of calcium flux? Sleep Res 18:78

Mendelson W, Skolnick P, Martin J, Luu M, Wagner R, Paul SM (1984): Diazepam-stimulated increases in the synaptomal uptake of 45Ca: Reversal by dihydropyridine calcium channel antagonists. Eur J Pharmacol 104:181-183

Merlotti L, Roehrs TA, Sicklesteel J, Kaffeman M, OchsCampbell R, Wittig R, Roth T (1992): Sedative and performance effects of hypnotics. Sleep Res 21:64

Nicholson AN, Stone BM (1986): Antihistamines: Impaired performance and the tendency to sleep. Eur J Clin Pharmacol 30:27-32

Rechtschaffen A, Kales A (1968): A Manual of Standardized Terminology, Techniques, and Scoring System for Sleep Stages of Human Subjects. Los Angeles, BIS/BRI, UCLA

Roehrs T, Zwyghuizen-Doorenbos A, Roth T (1989): Sleep extension, enhanced alertness and the sedating effects of ethanol. Pharmacol Biochem Behav 34:321-324

Roehrs T, Zwyghuizen-Doorenbos A, Roth T (1993): Sedative effects and plasma concentrations following single doses of triazolam, diphenhydramine, ethanol, and placebo. Sleep 16:301-305

Roehrs T, Tietz E, Zorick F, Roth T (1984): Daytime sleepiness and antihistamines. Sleep 7:137-141

Roehrs T, Zwyghuizen-Doorenbos A, Zwyghuizen H, Roth $T$ (1990): Sedating effects of ethanol after a nap. Alcohol Drugs Driving 6:351-356

Roehrs T, Zwyghuizen-Doorenbos A, Smith D, ZorickF, Roth $T$ (1988): Reversal by caffeine of triazolam-induced impairment of waking function. In Hindmarch $\mathrm{I}$, Ott $\mathrm{H}$ (eds), Benzodiazepine receptor Ligands, Memory, and Information Processing. Berlin, Springer-Verlag, pp 194-201

Schwartz JC, Barbin G, Duchemin AM, Garbarg M, Llorens C, Pollard H, Quach TT, Rose C (1982): Histamine receptors in the brain and their possible functions. In Ganellin CR, Parsons ME (eds), Pharmacology of Histamine Receptors. Boston, Wright PSG, pp 351-391

Zwyghuizen-Doorenbos A, Roehrs T, Lamphere J, Zorick F, Roth T (1988): Increased daytime sleepiness enhances ethanol's sedative effects. Neuropsychopharmacology $1: 279-286$ 